\title{
Optimum Focal Length Range For Thin-Lenses Optical Arrays Having Spatial Integration Feature
}

\author{
Hala Kamal ${ }^{*}$, Daniel V'azquez and Javier Alda \\ Optics Department. University Complutense of Madrid. \\ School of Optics. Av. Arcos de Jal'on s/n. 28037 Madrid. Spain.
}

\begin{abstract}
A simple realization of a spatial integrator array is constructed by using individual units composed of two thin lenses. The spatial integration feature depends on the value of the focal distance of one of the lens. This focal length and its relation with the thickness of the individual element are related with the lateral magnification defining the synthetic image given by the array. Although the focal length of the other lens can be arbitrarily selected, we have analyzed the optimum range of focal distances that increases the amount of energy falling on the synthetic image. This analysis has been done for spatial integrator arrays having spherical dome configuration and planar configuration. Numerical analysis of the behavior of the optimized units, performed in a meredional plane, is presented. The level the total irradiance reaching the synthetic image plane and the uniformity of the irradiance distribution on it have been measured. The coefficient of uniformity as well as the image width have been evaluated and compered. The results clearly demonstrate that the performance of the optimized unit is largely improved relative to the non-optimized one. The study has been done both in the paraxial range and by using real ray-tracing tools. Comparison of the paraxial calculation of the spatial integrator array and those obtained by real raytracing enables the practical choice of the upper limits of the focal length range in order to avoid aberrations effects and significant deviation from the paraxial behavior situation.
\end{abstract}

\section{Introduction:}

An optical array can be considered as a set of identical optical elements with similar characteristics located in some kind of regular misalignment and working in parallel (instead of the usual optical combinations where the individual systems work in series along the ray path). The framework of the

* On leave from Faculty of Science, Ain Shams University, Cairo, Egypt. 
theory of optical arrays was developed by Wang and Ronchi several years ago [1]. Recently it has been revisited and expanded by Wang and Zhao [2]. They present a matrix treatment carried out within the paraxial approximation. The design parameters of the array, as well as the geometrical parameters of its optical elements, determine the value of the determinant of the ray transfer matrix of the array. Accordingly, optical arrays may be classified with respect to the value of the determinant of their ray transfer matrices. This value defines whether an array is linear optical system or behaves non-linearly. One of the most interesting types which belong to the second category is the so called spatial integrator, or light integrator. Within the matrix optics framework, a spatial-integrator is an optical array with vanishing determinant, which produces a synthetic image at a given fixed plane, regardless the position, size or shape of the object in the object space. An optical array with spatial integration feature must fulfill the following condition,

$$
R=l-\frac{b}{d}
$$

where $R$ is the distance between the input plane of the array and the synthetic image plane, $l$ is the length of the individual optical unit, and $b$ and $d$ are the elements of the matrix of each unit of the optical array. The last equation indicates that the spatial integration feature doesn't depend on the $a$ and $c$ elements of the abcd matrix characterizing the individual optical element of the array. For a light integrator array composed of a pair of thin lenses or a thick lens element, it is clear that, the characteristics of the first element don't affect the integration feature.

Spatial integrators are not image-forming systems. They are intended to be used as collection optics in optical systems designed to produce a uniform distribution of irradiance at a given plane. This plane is named as the synthetic image plane. They may find applications in natural lighting systems, shadowless illumination systems, and in any other task where the detection of light sources, regardless of its position and size, within a given field of view is of interest. In some previous research we have developed several ideas for these kind of arrays [3-7]. The design of the array elements can be refined to optimize the transfer of radiant flux from the entrance plane to the synthetic image plane. This goal has been obtained for spatial integrators where the individual units are thick lenses [7]. In that case the array is better arranged in a spherical dome configuration. A planar array better suited to be integrated within architectural elements was proposed, fabricated and tested by some of the authors [6].

In the present work, we present a way to optimize spatial integrators fabricated with elemental units, configured as two thin-lenses combinations, 
both for spherical dome configuration and planar arrangement. An analytical solution is proposed and applied to obtain a range in the focal length of the first lens of the individual units. The obtained solution is numerically analyzed and the results show an improvement in the performance of the spatial integrator array. In Section 2 we present a simple method for designing optical arrays with spatial integration feature composed of elemental units having two thin lenses. The analytical and graphical result relates the three main parameters involved in the design; the thickness of the elemental unit, the desired size of the synthetic image, and the focal length of one of the thin lens used in every unit of the array. Section 3 and 4 present the solutions for optimizing the amount of radiant flux reaching the synthetic image plane, in the cases of spherical dome configuration and in the case of a planar arrangement, respectively. As far as our previous interest is focused in the planar spatial integrator we have made a numerical analysis of the behavior of the optimized units, performed in a meridional plane. Real ray-tracing software has also been used to numerically check the results obtained within the paraxial approach.

\section{Design of two Thin-Lenses Spatial Integrator Arrays:}

The optical axis of the individual units of a spatial integrator array intersect at a given point that defines the center of the synthetic image. The distance between the input surface of the optical array and the synthetic image plane is $R$, and it should be considered as the length of the optical array. This applies for spherical dome configuration arrays and planar arrays. In a spherical dome configuration the units of the light integrator array are arranged in such a way that the vertex of the input surface of each individual unit is located on a sphere of radius $R$ given by equation (1). As the paraxial approach neglects the axial displacement of the input plane of the individual misaligned optical unit, the input plane of each individual unit is coincident with the input plane of the array. In the planar arrangement case, the optical axis of the individual units is defined by joining the optical centers of the elements composing the individual units. They all intersect at the center of the synthetic image. The individual units of the type of light integrator array analyzed in this paper are formed by two thin lenses separated by a certain distance. Let $f{ }^{\prime}{ }_{1}$ and $f{ }^{\prime}{ }_{2}$ be the focal length of the first and second thin lens respectively, $h_{1}$ and $h_{2}$ their half transversal sizes, and $t$ is the distance between them. According to the matrix treatment, the ray transfer matrix representing the behavior of the individual unit between its input and output surfaces is given by,

$$
\mathbf{M}_{\text {unit }}=\left(\begin{array}{cc}
1 & 0 \\
-\frac{1}{f_{2}^{\prime}} & 1
\end{array}\right)\left(\begin{array}{ll}
1 & t \\
0 & 1
\end{array}\right)\left(\begin{array}{cc}
1 & 0 \\
-\frac{1}{f_{1}^{\prime}} & 1
\end{array}\right)=\left(\begin{array}{cc}
1-\frac{t}{f_{1}^{\prime}} & t \\
-\frac{1}{f_{1}^{\prime}}-\frac{1}{f_{2}^{\prime}}+\frac{t}{f_{1}^{\prime} f_{2}^{\prime}} & 1-\frac{t}{f_{2}^{\prime}}
\end{array}\right)
$$


The condition of vanishing determinant is satisfied when the distance between the input plane and the synthetic image plane obeys the following relation:

$$
R=t\left(1-\frac{f_{2}^{\prime}}{f_{2}^{\prime}-t}\right) .
$$

This equation is the first design condition for light integrator array built up with two thin lenses. Normalizing all lengths with respect to $R$ we get

where,

$$
\mu_{2}=\mu_{\mathrm{t}}\left(1-\mu_{\mathrm{t}}\right),
$$

$$
\mu_{2}=\frac{f_{2}^{\prime}}{R} \text {, and } \quad \mu_{t}=\frac{t}{R} .
$$

For optical arrays with spatial integration feature, the lateral magnification of the array is defined as the ratio between the synthetic image size and the transversal size of the input pupil of the element; this is because the input pupil and the synthetic image are conjugated elements through the second lens. For thin lens light integrator array, the modulus of the transverse magnification, $|M|$, in terms of the previously defined normalized variables is given by,

$$
|M|=\frac{1-\mu_{t}}{\mu_{t}} .
$$

The value of $t$ changes from zero to $R$ ( $\mu_{t}$ ranges from zero and one). Fig. (1), represents the conditions for designing arrays with spatial integration features. The parameter $\mu_{2}$ and the modulus of the lateral magnification of the array, $|M|$, are given as a function of the thickness parameter, $\mu_{t}$. For example, if the desired magnification is set, it will provide a value of the thickness of the unit of the array and a corresponding value of the focal length of the second unit. Typically, a spatial integrator produces a synthetic image that is larger than the lateral size of the first element of the array. Therefore, the lateral magnification will be larger than 1 and the region of interest in the diagram is restricted to $\mu_{t}<$ 0.5 . The maximum value of $\mu_{2}$ is equal to 0.25 , which means that the value of $f_{2}$ should be chosen to be at least four times smaller than the length of the array. This result is in perfect accordance with the minimum object-image distance for real object and real image conjugation, and corresponds with the location of the anti-principal planes of the second lens. 


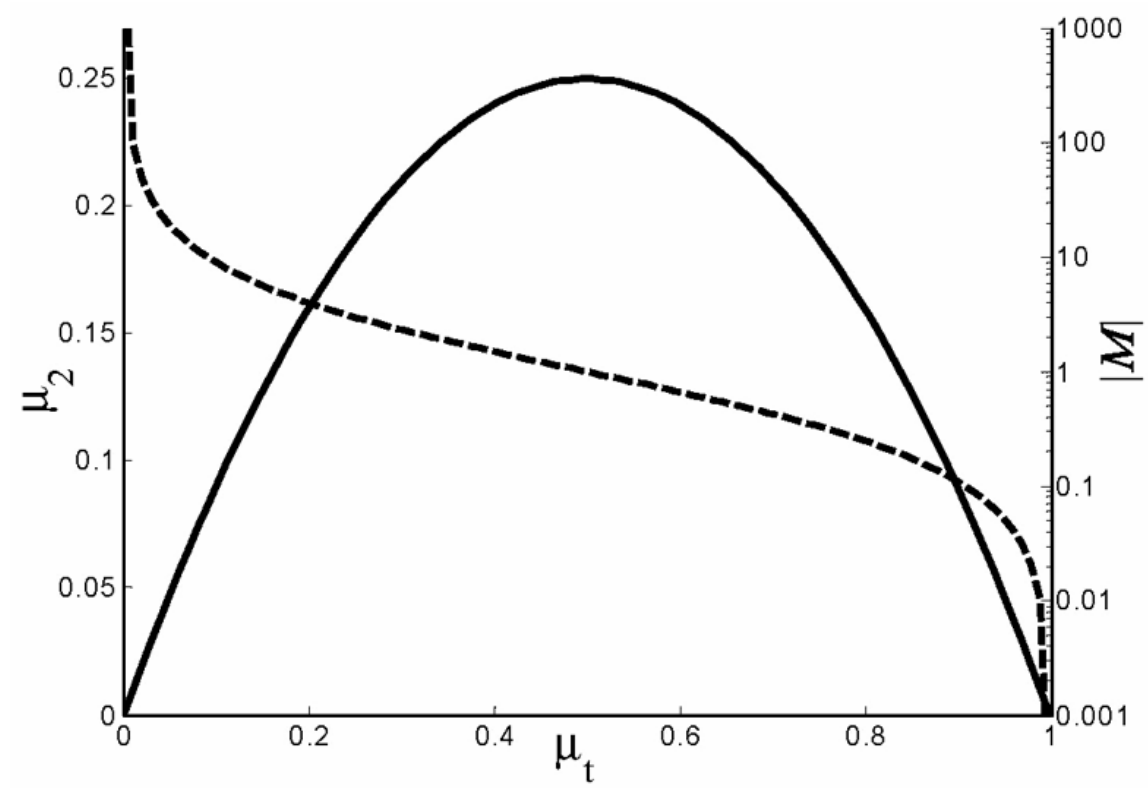

Fig. (1): Relation between the focal length of the second lens $f_{2}$ (represented by $\mu_{2}$ $=\left(f{ }_{2} / \mathrm{R}\right)$ and the individual unit size $t$ (represented by $\left.\mu_{\mathrm{t}}=\mathrm{t} / \mathrm{R}\right)$. The dashed line corresponds to the modulus of the lateral magnification, plotted in log-scale, as a function of the individual unit size.

\section{Spatial Integrator With Spherical Dome Configuration:}

In this section we optimize each unit of an optical array configured with its elements on a spherical dome. The optimization avoids the energy loss through its walls and therefore it transfer as much energy as possible from the input surface of the array onto the synthetic image plane. The calculus is done under the paraxial approach. The paraxial ray-tracing of the optimization conditions is plotted in Fig. (2)a.

As the first element of the individual optical unit doesn't affect the integration feature, we can modify its focal length without changing the array behavior. For every array unit located at the position $\mathrm{k}$ the first thin-lens has an

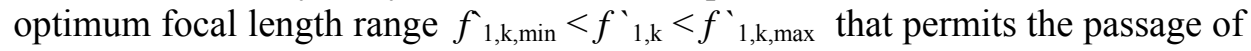
all light rays incident on its surface to go through the second thin-lens and finally contribute to form the synthetic image. The value of $k$ labels the individual unit according to its position within the array. Considering a parallel bundle of rays incident on the array surface and misaligned an angle $\delta$ with respect to the axis of the array (the axis of the array is considered as its axis of symmetry). The maximum focal length, $f^{{ }^{\prime}}{ }_{1, k, \max }$, is defined by the light ray incident on the first lens at the maximum design height, $h_{1}$, reaching the second 
lens at its maximum height, $h_{2}$. This value can be calculated by fixing the ray's slope after the first lens equal to half the angle subtended by the individual unit at the maximum height allowed by the first lens, $\varphi$, (this angle coincides with the half-angle subtended by the individual unit from the center of the synthetic image). This condition can be written in a matricial form as;

$$
\left(\begin{array}{c}
h_{1} \\
-\phi
\end{array}\right)=\left(\begin{array}{cc}
1 & 0 \\
-\frac{1}{f_{1, k}^{\prime}, \max } & 1
\end{array}\right)\left(\begin{array}{c}
h_{1} \\
\omega_{k}+\delta
\end{array}\right)
$$

where $\omega_{\mathrm{k}}$ is the slope of the incident ray with respect to the axis of the individual element, $\delta$ is the slope of the ray with respect to the axis of the array, and $\mathrm{f}_{1, \mathrm{k} \text {,max }}>t$. On the other hand, the minimum value of the first lens focal length $f_{1, k, \min }$ is optimized by considering the light ray incident at the minimum height of the lens to reach the second lens at its maximum height. This condition can be written in a matricial form as,

$$
\left(\begin{array}{c}
-h_{1} \\
\sigma
\end{array}\right)=\left(\begin{array}{cc}
1 & 0 \\
-\frac{1}{f_{1, k, \min }^{\prime}} & 1
\end{array}\right)\left(\begin{array}{c}
-h_{1} \\
\omega_{k}+\delta
\end{array}\right)
$$

where $\sigma=\left(h_{1}+h_{2}\right) / t$ is the slope of the refracted ray and $\quad f^{\prime}{ }_{1, \mathrm{k}, \min }<t$.

Two configurations are possible for this type of optical array. The first configuration considers a central unit whose axis coincides with the array axis figure. In this case the inclination of the unit with the array axis according to its order is given by, $\omega_{\mathrm{k}}=2 k \varphi$ where $k=0, \pm 1, \pm 2, \ldots, \pm n$ is the individual unit order. The zero order corresponds to the central element while $n$ is the order of the outset element of the array. As a consequence of equations (7) and (8), the optimum focal length range of the first lens corresponding to each array unit and according to its order is given by,

$$
\frac{h_{1}}{\sigma-\omega_{k}}<f_{1, k}^{\prime}<\frac{h_{1}}{\phi+\omega_{k}},
$$

where we have assumed that $\delta=0$. As $f^{{ }^{\prime}}{ }_{1, \mathrm{k}, \min }$ is smaller than $f^{{ }^{\prime}{ }_{1, \mathrm{k}, \max } \text {, the }}$ maximum order of the unit should obey the following relation,

$$
n<\frac{R\left(h_{1}+h_{2}\right)+t h_{1}}{4 t h_{1}} .
$$


Therefore, the maximum number of units that will contribute to form the synthetic image can be deduced as,

$$
N=1+2 \times \text { Previous integer }[n] .
$$

The second configuration has not one central element. In this case all array units are off-axis with respect to the longitudinal axis of the array. The slope of the incident ray corresponding to the individual unit order becomes

$$
\omega_{k}=\left(2 k-\frac{k}{|k|}\right) \phi
$$

where $k= \pm 1, \pm 2, \ldots, \pm n$ is the unit order. The first unit above the array axis has $k=1$, while $k=-1$ corresponds to the first unit below the array axis.

\section{Spatial integrator with plane configuration:}

In the previous section the units of the light integrator array were arranged in a spherical configuration. Alternatively, a plane light integrator array has been proposed [6]. Now the vertexes of the individual units are located on a plane. This plane arrangement has the advantage to be easily integrated. The individual units of the light integrator array are built up by a couple of thin lenses. The focal length of the second unit has to comply with the conditions expressed in Section 2. The optical axis of each unit is the line joining the centers of each thin lens in that unit. Each unit has its own optical axis. All intersects at the centre of the synthetic image plane.

In the present work, the plane light integrator array is composed of two plane positive thin lens arrays (PTLA) separated by a distance $t$ (see figure (2)b. The first thin lens array, PTLA1, is considered to be the input surface of the light integrator array and the second thin lens array, PTLA2, is its output surface. The elements of PTLA2 are of identical focal length, $f_{2}{ }_{2}$, to comply with the vanishing determinant condition (equation 1). However, the elements of PTLA1 are not identical. The individual elements of PTLA1 are optimized to avoid energy losses through the walls of the individual units of the light integrator array and to transfer all light energy incident on PTLA1 to the synthetic image plane. 


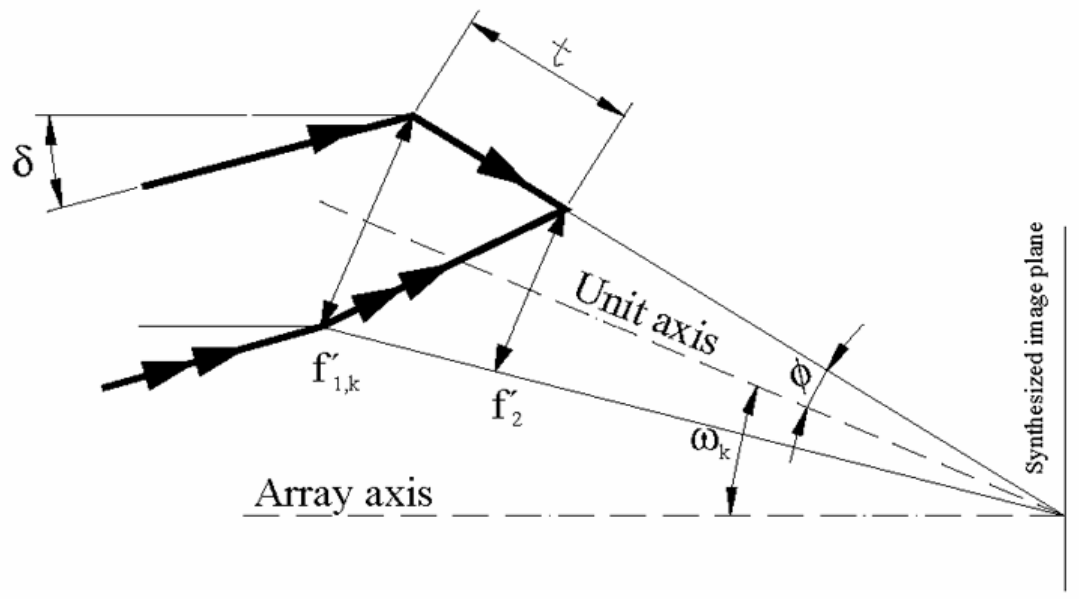

(a)

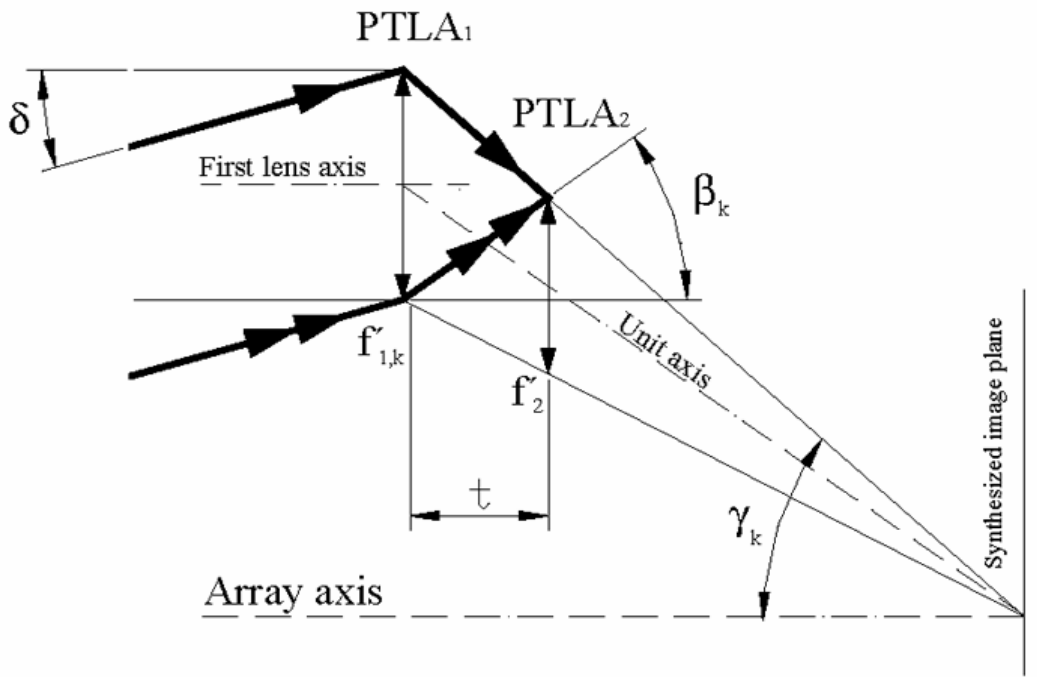

(b)

Fig. (2): Scheme of two thin-lens light integrator arrays. (a) Spherical dome configuration. (b) Plane configuration.

The goal of this section is to find the optimum focal length range for PTLA1 in case of planar light integrators. As a consequence of the plane arrangement the angle that subtends each individual unit is not equal; instead it becomes smaller as we move away from the array axis. In the analysis we begin with a parallel bundle of rays falling on the optical array with an angle $\delta$ with the array axis. The maximum value of the focal length range, $f^{{ }_{1}, k, \max }$, corresponding to the $k^{\text {th }}$ unit can be calculated by considering the light ray incident on the maximum height of the first lens to be deviated to an angle, $\gamma_{k}$, Figure (2)b. This condition can be written in a matricial form as, 


$$
\left(\begin{array}{c}
h_{1} \\
-\gamma_{k}
\end{array}\right)=\left(\begin{array}{cc}
1 & 0 \\
-\frac{1}{f_{1, k, \max }^{\prime}} & 1
\end{array}\right)\left(\begin{array}{l}
h_{1} \\
\delta
\end{array}\right) .
$$

Where, $\gamma_{\mathrm{k}}=\left(2 k h_{1}\right) / R$, and $k= \pm 1, \pm 2, \ldots, \pm n$. Accordingly,

$$
f_{1, k, \max }^{\prime}=\frac{h_{1}}{\gamma_{k}+\delta} .
$$

The minimum value of the focal length range, $f^{{ }^{\prime}}{ }_{1, \mathrm{k}, \min }$, corresponding to the $k^{\text {th }}$ unit can be calculated by considering the light ray incident at the minimum height of the first lens to be deviated to an angle $\beta_{\mathrm{k}}$ (see Fig. (2)b. This can be written in a matricial form as,

$$
\left(\begin{array}{c}
-h_{1} \\
\beta_{k}
\end{array}\right)=\left(\begin{array}{cc}
1 & 0 \\
-\frac{1}{f_{1, k, \min }^{\prime}} & 1
\end{array}\right)\left(\begin{array}{c}
-h_{1} \\
\delta
\end{array}\right),
$$

where, $\beta_{\mathrm{k}}=\left(2 h_{1}-2 m\left(h_{1}-h_{2}\right)\right) / t$, and $k= \pm 1, \pm 2, \ldots, \pm n$. Accordingly,

$$
f_{1, k, \min }^{\prime}=\frac{h_{1}}{\beta_{k}-\delta} .
$$

For a parallel bundle of rays aligned with the array axis, $\delta=0$, the optimum first thin lens focal length range for a planer thin lens light integrator array becomes.

$$
\frac{h_{1}}{\beta_{k}}<f_{1, k}^{\prime}<\frac{h_{1}}{\gamma_{k}}
$$

Substituting for $\beta_{\mathrm{k}}$ and $\gamma_{\mathrm{k}}$ we get,

$$
\frac{h_{1} t}{2 h_{1}-2 k\left(h_{1}-h_{2}\right)}<f_{1, k}^{\prime}<\frac{R}{2 k}
$$

The maximum value of the focal length range corresponding to each unit order is greater than the separation between the two thin lenses units and smaller than the distance between the input plane and the synthetic image plane. Besides, the minimum focal length is smaller than the array thickness $t$. Also, it is found that $f^{{ }^{\prime}}{ }_{1, k, \max }$ depends on the array parameter $R$, while $f^{{ }^{\prime}}{ }_{1, k, \min }$ is a function of the individual unit parameters. For a planar light integrator array 
with a defined parameters $h_{1}, h_{2}, t$ and $R$. The maximum order of the element is that integer, which obeys the following condition,

$$
n<\frac{h_{1}}{2\left(h_{1}-h_{2}\right)} \text {. }
$$

The maximum number of units that can be arranged around the array axis for an array with no central element is;

$$
N=2 \times \text { Previous integer }[n] .
$$

This equation is also applicable for spherical dome configurations with no central elements. At the same time, equation (11) would be applicable using the value of $\mathrm{n}$ given in equation (19), when no central element is present in an optical array with spherical dome configuration.

\subsection{Synthetic Image Calculation:}

In order to understand and clarify the meaning of the previously obtained relations we have performed numerical simulations for a planar spatial integrator composed of two lenses. The values of the focal length $f^{\prime}{ }_{2}, t$, and $R$, used in the numerical calculation are given in Table (1). When applying the conditions for spatial integration feature described in section 2 , the value of the focal length $f^{\prime}{ }_{2}$ is $140 \mathrm{~mm}$, that differs slightly from the value considered in Table (1). This is because $f^{{ }^{\prime}}{ }_{2}=137.9 \mathrm{~mm}$ is the focal length of the ophthalmic lenses used for the practical realization of a plane spatial integration arrays fabricated by using ophthalmic "off-the-shelf" lenses having a power of $F_{2}{ }_{2}=$ $7.25 \mathrm{D}$. The manufacturing tolerances of the lenses made possible to consider the optical array having an spatial integration feature, as it was demonstrated in a previous contribution [7]. The calculation made in this paper shows an improvement of the radiometric performance of this array. In Fig. (3) we have sketched the analyzed array. In this case we have assumed that the units are placed side-by-side without intermediate blank spacers. The focal length of the elements of PTLA2 is the same for all the units. However, the focal length, $f^{\prime}{ }_{1}$, of PTLA1 are chosen to be within the range calculated in this section and expressed in Table (2). They are different for each location of the array (although preserving the symmetry with respect to the optical axis of the array). In Fig. (4), we have plotted the transmittance at the input plane of the array for three different designs. Cases (a) and (b) correspond to the minimum and maximum values of the focal length, $f^{{ }}{ }_{1}$, respectively. These results can be compared with case (c) where no lens is placed (infinite focal length). In the later case, the transmittance is zero for some regions of the entrance plane, even for the first unit of the array. Therefore, the efficiency in the transfer of flux is 
lower than those in the optimized cases (a) and (b), notice that the transmittance values are plotted within a narrow range of about $2 \%$ variation.

Table (1): Geometrical parameters of a planar thin lens light integrator array. The refractive index of the lenses material is 1.523 .

\begin{tabular}{|c|c|}
\hline$R$ & $1280 \mathrm{~mm}$ \\
\hline$f_{2}$ & $137.9 \mathrm{~mm}$ \\
\hline$h_{1}$ & $40 \mathrm{~mm}$ \\
\hline$h_{2}$ & $35 \mathrm{~mm}$ \\
\hline$t$ & $160 \mathrm{~mm}$ \\
\hline
\end{tabular}

Table (2): The optimum minimum and maximum values of the first thin lens focal length corresponding to each unit order for a planar thin lenses light integrator array with geometrical parameters given in Table 1.

\begin{tabular}{|c||c|c|}
\hline Order of the unit, $k$ & $f^{{ }^{\prime}{ }_{1, \mathrm{k}, \min }}$ & $f^{{ }^{\prime}{ }_{1, \mathrm{k}, \max }}$ \\
\hline 1 & $91.4 \mathrm{~mm}$ & $640 \mathrm{~mm}$ \\
\hline 2 & $106.7 \mathrm{~mm}$ & $320 \mathrm{~mm}$ \\
\hline 3 & $128 \mathrm{~mm}$ & $213.3 \mathrm{~mm}$ \\
\hline
\end{tabular}

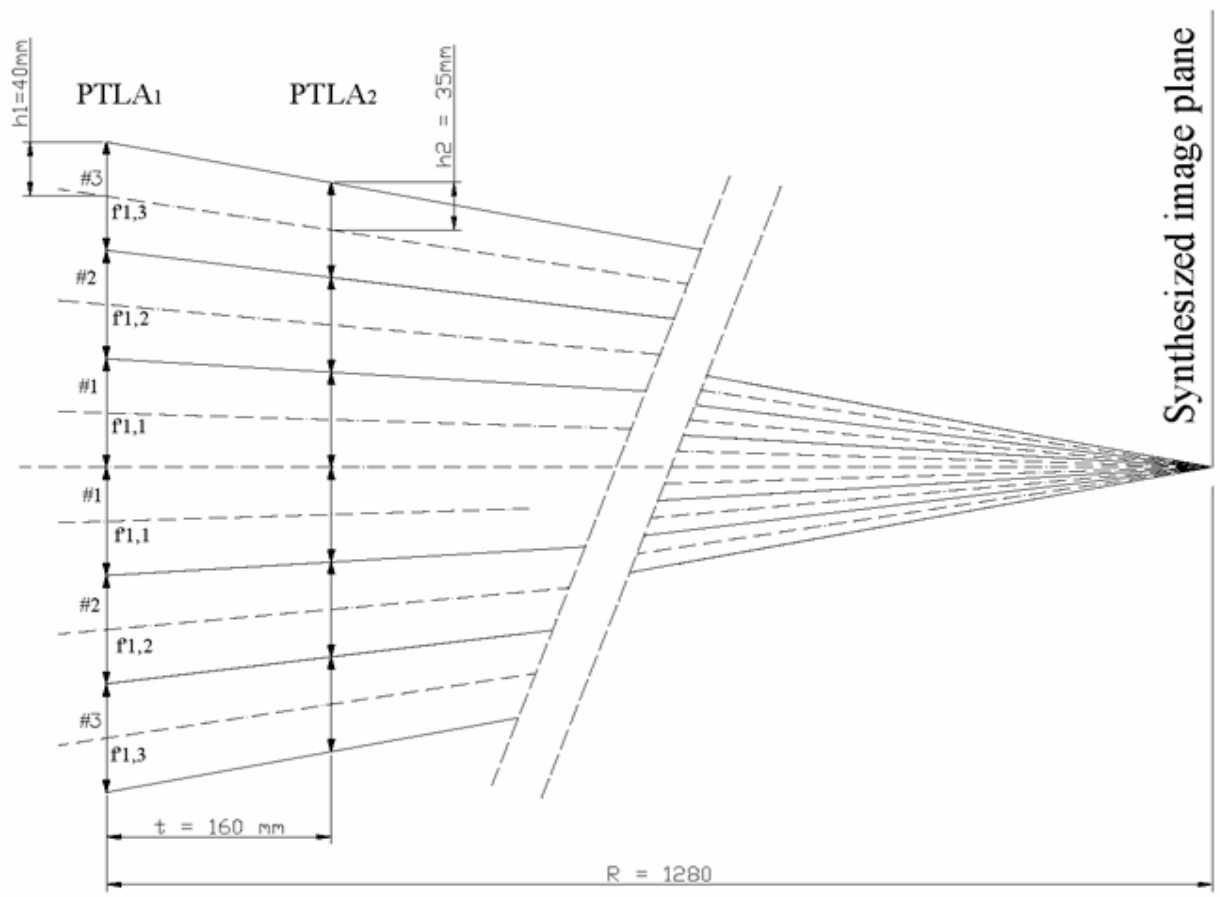

Fig. (3): Plot of the optical array having a plane configuration. The values of the parameters are given in Table (1). The optimum values for $f_{1, \mathrm{k}}$ are in the range given in Table(2). 

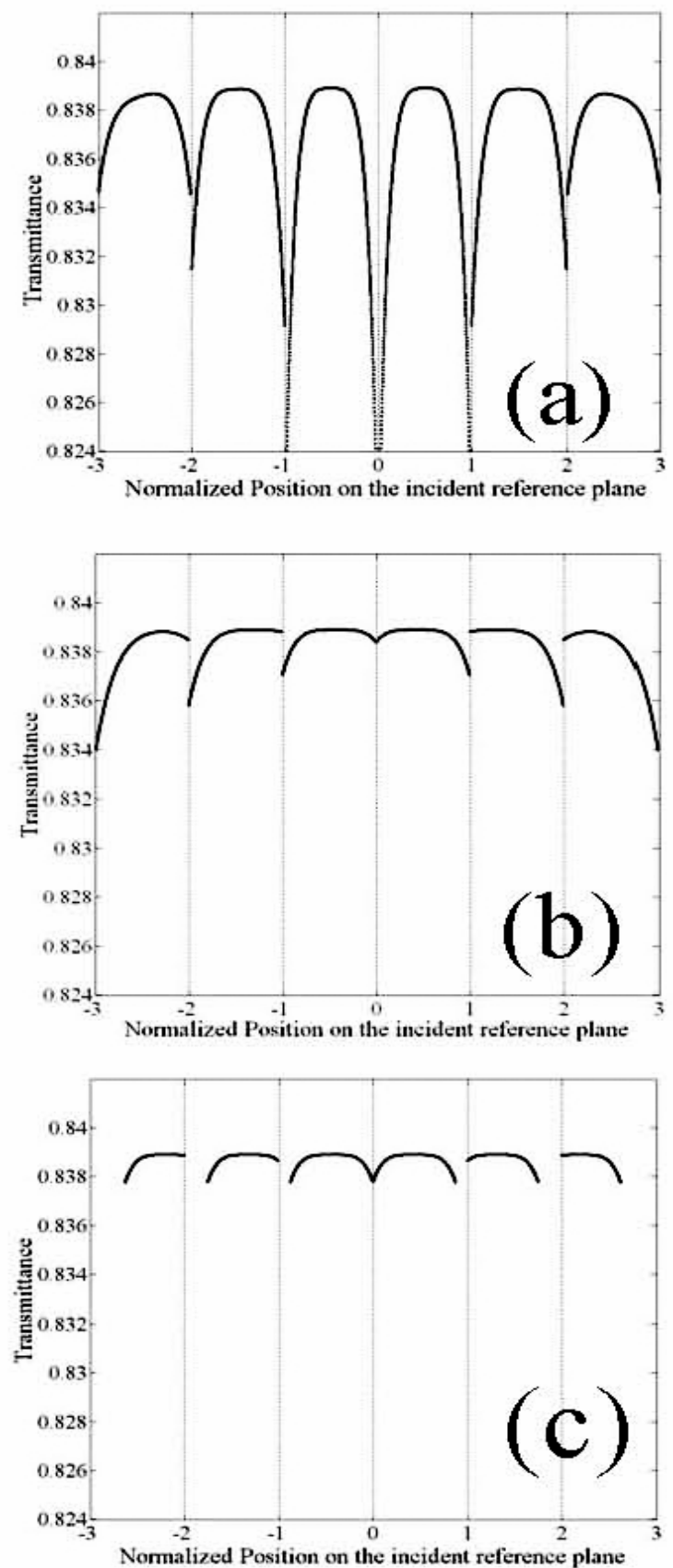

Fig. (4): Transmittance of the array on the input reference plane (the pupil functions of the optical array), for three different designs, (a) and (b) correspond to minimum and maximum values of $f^{\prime}{ }_{1}$, while (c) corresponds to infinite focal length. 
The transmittance calculation has been done by assuming a bi-convex thin lens format both in PTLA1 and PTLA2, and taking into account the Fresnel transmission coefficients for unpolarized light. After propagating the light to the synthetic image plane, it is possible to obtain the energy distribution on that plane. This is done in Fig. (5).a for the same three cases where the rays entering the system at PTLA1. All the rays reach the synthetic image plane when the optimization is applied. In addition, related with this result, we have found that the insertion of lenses at the PTLA1 having focal lengths properly optimized, adds an amount of $32 \%$ of energy at the synthetic image plane (with respect to the case of no lenses placed at PTLA1). Fig. (5)b shows the irradiance distribution obtained along the direction where the array units is arranged. These results are obtained by using a real ray-tracing software (TracePro). The figure has been scaled to make possible a comparison with the paraxial results presented in Fig. (5)b. The dashed line is for the minimum focal length solution, the solid line applies to the maximum focal length, and the dotted line corresponds to an optical array without lenses on the first input surface.
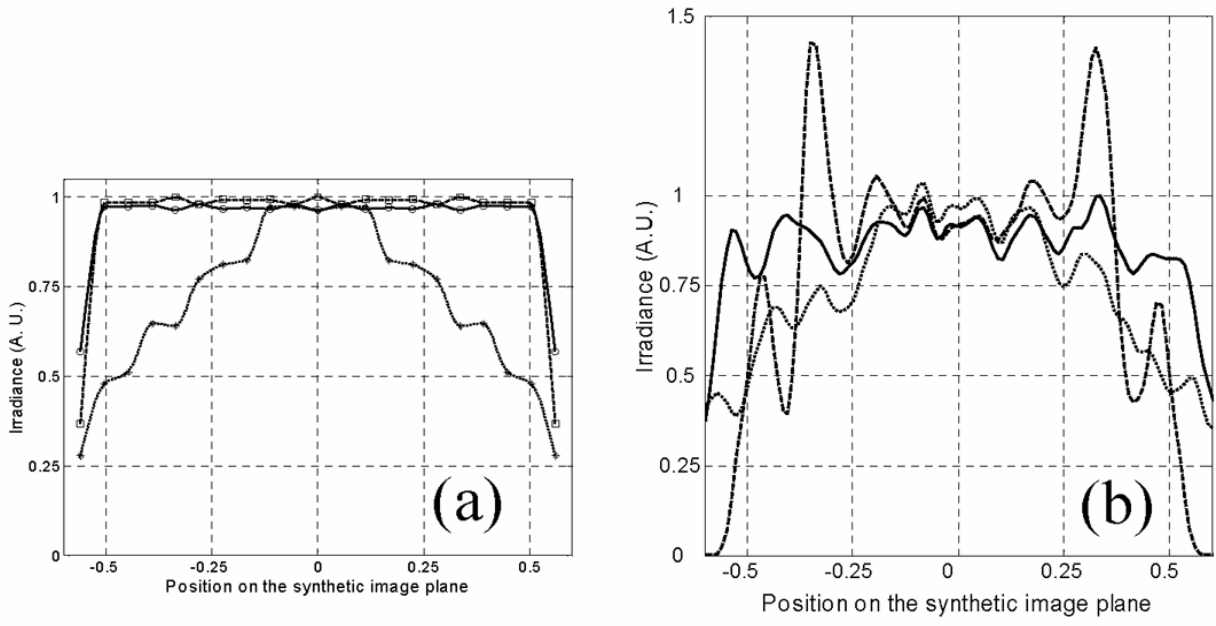

Fig. (5): a) Irradiance distribution (in arbitrary units) onto the synthetic image plane, obtained by paraxial calculation, for three different focal length values of

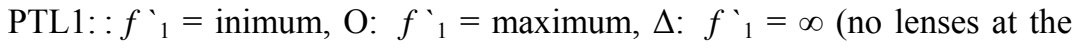
input plane).

b) Irradiance distribution obtained along the direction where the array units is arranged obtained by ray-tracing. The figure has been scaled to make possible comparison with the paraxial results. Dashed line : minimum focal length, solid line: maximum focal length, and the dotted line corresponds to an optical array without lenses on the first input surface.

If no lenses are placed on the PTLA1 the uniformity of the synthetic image plane is degraded. The cause of this degradation is related with the 
existence of zero-transmittance regions in the entrance plane of the optical array as it was shown in Fig. (4). The graphical results obtained here are also parameterized by calculating a coefficient of uniformity $u$ for the energy distribution, $\Phi(\mathrm{x})$, defined as

$$
u=\int_{\text {paraxial }}\left|\frac{\partial \Phi(x)}{\partial x}\right| d x .
$$

This parameter is zero for a perfect uniform distribution within the paraxial synthetic image (please note that the integration is made only on the spatial region of the paraxial synthetic image plane). The values of $u$ for the optimized cases are lower than the value obtained for the case without lenses at PTLA1. On the other hand, we have evaluated the image width defined as

$$
\omega=2 \sqrt{\frac{\int x^{2} \Phi(x) d x}{\int \Phi(x) d x}} .
$$

These results can be compared to the image width value obtained for an ideal rectangular energy distribution having a size equal to the paraxial synthetic image size, $\omega=0.623$. In Table (3), we can check that those values corresponding to the optimized cases are closer to the ideal one than the value obtained for the non-optimized case.

Table (3): The image width (related with the 2nd order moment) of the energy distribution, the coefficient of uniformity, the average transmittance and the percentage of outgoing rays to incoming rays.

\begin{tabular}{|c||c|c|c|c|}
\hline $\begin{array}{c}* \\
\text { Case }\end{array}$ & $\begin{array}{c}\text { Image width } \\
\omega\end{array}$ & $\begin{array}{c}\text { Coeff. of Uniformity } \\
u\end{array}$ & $\begin{array}{c}\text { Average } \\
\text { transmittance }\end{array}$ & $\begin{array}{c}\text { Percentage of outgoing } \\
\text { rays }\end{array}$ \\
\hline $\mathrm{a}$ & 0.634 & $0.162 \%$ & 0.8369 & 100 \\
\hline $\mathrm{b}$ & 0.641 & $0.148 \%$ & 0.8383 & 100 \\
\hline $\mathrm{c}$ & 0.571 & $1.035 \%$ & 0.6288 & 75 \\
\hline
\end{tabular}

* (a) A planar thin lens light integrator array with geometrical values given in Table (1) and having a PTLA1 with $f^{{ }^{\prime}}{ }_{1, k, \min }$, Case (b) with $f^{{ }^{\prime}}{ }_{1, \mathrm{k}, \max }$ and case (c) is an array with $f^{{ }^{\prime}}{ }_{1, \mathrm{k}}=\infty$.

The results computed in a meridional plane are also checked by calculating the two dimensional irradiance distribution at the synthetic image plane by using Trace Pro software. 8 The modelized system is a collection of six units along Y direction. The output of TracePro is also plotted in Fig. (6) for the three previously considered cases. A profile of the spatial distribution along $\mathrm{Y}$ direction at $\mathrm{x}=0$ is also given in Fig. (5)b, (this figure has been deformed for 
better comparison with the paraxial calculation presented in Fig. (5)a. Some remarks can be made from the results produced by Trace Pro. First of all we can see that the case of $f_{1, k, \min }$ is quite different than in the paraxial case. This is due to the effect of the optical aberration produced by the first lens. From Table (2), we can see that the F\# of these lenses is quite extreme $(1.1,1.3$, and 1.6 for $k=1,2$, and 3 respectively). Therefore, aberrations are at work and make the system perform quite differently than its paraxial counterpart. This behavior suggests another practical consequence: Once the focal range for $f^{\prime}{ }_{1, \mathrm{k}}$ is obtained, the larger value of the range will be preferred to the lower value. At the same time, these lenses would be cheaper and easier to be fabricated than those selected in the case of $f_{1, k, \mathrm{~min}}$.

The two dimensional irradiance distribution for three optical arrays composed of six elemental units fabricated using the values presented in Table (2) is illustrated in Fig. (6). Curves $a$ and $b$ correspond to the minimum and the maximum values of the focal distances, $f^{{ }_{1, k, m i n}}$. and $f^{{ }_{1, k, m a x}}$ respectively. Curve c correspond to $f^{\prime}{ }_{1, \mathrm{k}}=\infty$. The white circle corresponds to the size of the paraxial synthetic image. The uniformity is better for case (b) in accordance with the uniformity parameter calculated within the paraxial approach and presented in Table (3). The real ray-tracing results also demonstrate that the synthetic image is more uniform and brighter when using an optimized solution (compare Figures (6)b and (6)c).

\section{Conclusions:}

An optimized spherical thin lens light integrator array is proposed. An analytical and graphical solution for the design of this type of spatial integrator is presented. It relates the dimensional and optical parameters characterizing the spatial integrator: the size of the synthetic image with respect to the transversal size of the input plane of the individual unit, the focal length of the second thin lens used in the unit, and the thickness of the array. The analysis is made by using variables normalized to the total distance between the entrance plane and the synthetic image plane. The focal length of the first thin lens of the array is left as a free parameter that does not affect the spatial integration feature. Then, it can be used to improve the performance of the optical array as a system devoted to the transfer of flux between the entrance plane and the synthetic image plane. 


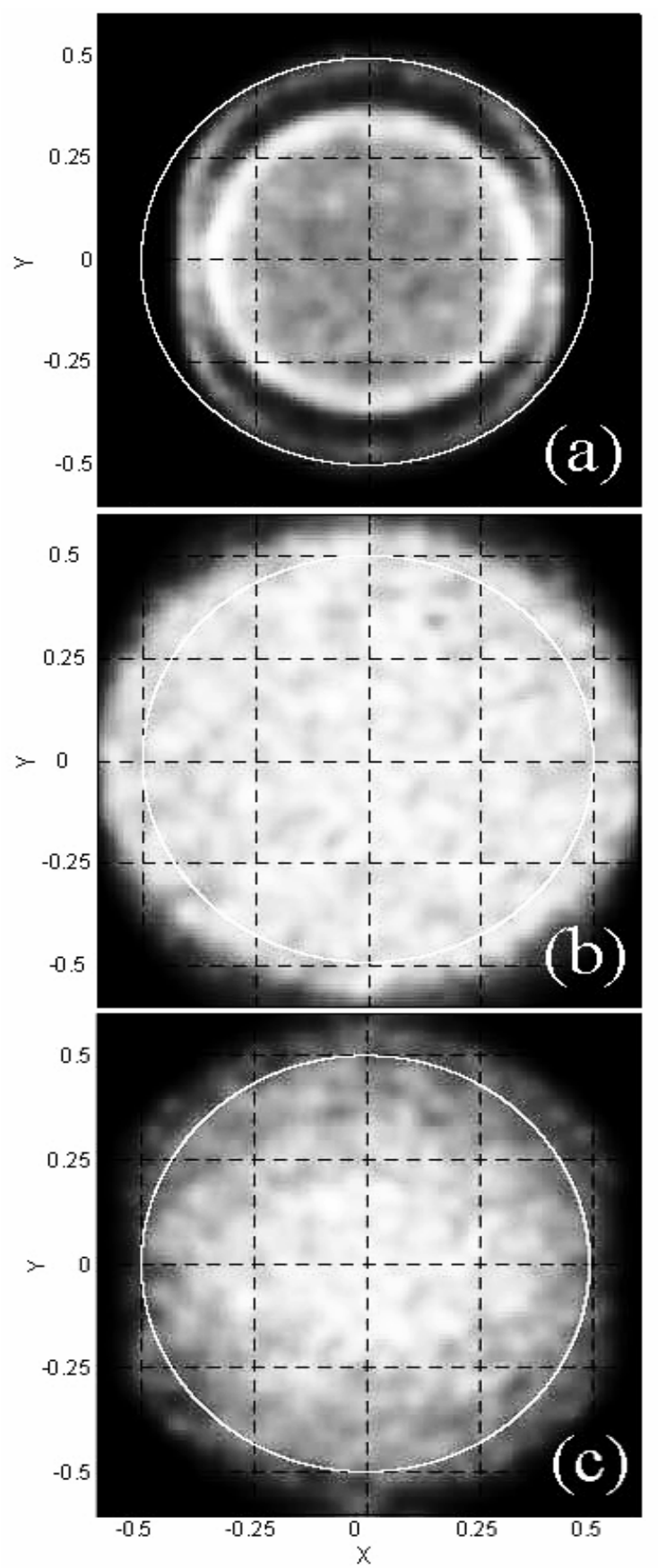

Fig. (6): Two dimensional irradiance distribution for three optical arrays composed of six elemental units fabricated using the values presented in Table 2. a for

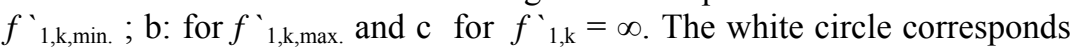
with the size of the paraxial synthetic image. 
To obtain the focal length of the first thin lens we force all the arrays incoming to the first lens to reach the second lens aperture. Those rays passing through the second lens will also reach the synthetic image plane. This condition also precludes the loss of energy when light falls on the lateral walls of the units, or cross-talking between adjacent units when no lateral walls are separating the units. Matricial optics relations are used to perform such optimization. The calculus is made for two different arrangements: the spherical dome configuration and the planar one. The obtained results shows that each array unit, depending on its position within the optical array, has an optimum focal length range. The existence of such a range may be used to properly choose lenses easily available, reducing the cost of the fabricated array. The numerical simulations made on the proposed optimized designs clearly demonstrate that the synthetic image is more uniform and brighter. An increments of about $32 \%$ in the total irradiance reaching the synthetic image plane, is achieved, when using lenses with optimized focal lengths. The results provided from the paraxial calculation of the spatial integrator array have been compared with those obtained by using a real ray-tracing software package. An important consequence of this comparison has been the practical choice of the upper limit of the $f_{1, \mathrm{k}}$ range in order to avoid the use of extreme numerical apertured lense that may include aberrations and significant deviations from the paraxial behavior situation.

\section{Acknowledgments:}

This work has been partially supported by the Ministerio de Ciencia y Tecnolog' 1 a of Spain, under the project TIC2001-1259.

\section{References:}

1. S. Wang, L. Ronchi, "Principles and design of optical arrays", Progress in Optics, pp. 279-347, North-Holand, Amsterdam (1988).

2. S. Wang, D. Zhao, "Matrix optics", Springer-Verlag, Heidelberg, (2000).

3. J. Alda, H. Kamal, E. Bernabeu Opt. Eng., 36, 2872 (1997).

4. H. Kamal, "Design and properties of optical arrays", $\mathrm{PhD}$ dissertation, Universidad Complutense de Madrid, Spain, 1998, (in English).

5. D. Vázquez, E. Bernabeu, Lig. Res. Tech., 29, 33 (1997).

6. D. Vázquez, J. Alda, and E. Bernabeu, Appl. Opt., 38, 1133 (1999).

7. H. Kamal, Optik, 114, 213 (2003).

8. Trace Pro is a product of Lambda Research Corporation. Littleton, MA 01460-4400, USA. http://www.lambdares.com/products/tracepro/ index.phtml (accessed January, 2004) 